\title{
Traps, Pitfalls and Misconceptions of Machine Learning applied to Scientific Disciplines
}

\author{
Davide Del Vento \\ ddvento@ucar.edu \\ Computational and Information System Lab \\ National Center for Atmospheric Research \\ Boulder, Colorado
}

\author{
Alessandro Fanfarillo \\ elfanfa@ucar.edu \\ Research Applications Lab \\ National Center for Atmospheric Research \\ Boulder, Colorado
}

\begin{abstract}
In the last decade, Machine Learning has experienced a dramatic increase in performance on a wide variety of tasks, including computer vision, speech recognition, text parsing, and language translation, just to name a few. This has corresponded to an understandable hype especially for the remarkable results achieved in some cases. Therefore, practitioners of Scientific Disciplines have become interested in utilizing new Machine Learning techniques, and have sometimes started doing so with mixed success.

The purpose of this paper is to describe some of the common Traps, Pitfalls and Misconceptions of Machine Learning as relevant to the Scientific Discipline, and how to avoid them. In fact, Machine Learning and Deep Learning are fast evolving fields, and some of the astonishing results achieved recently sit on small but important details which have become the state of the art. Some of these details are not broadly known by the scientific community. No new scientific result is presented in this paper, which is a survey and a summary of the best of the field, for the benefit of researchers with limited experience.

It is not the intention of the authors to provide any criticism to the work of experienced practitioners, particularly not to the ones working on the cutting edge of what is currently possible: in these cases expert researchers may well be doing exactly what we recommend here to avoid, and for a good reason. However we believe that the advice provided here will be useful, and perhaps even a reference, for the newcomers of the field.
\end{abstract}

\section{ACM Reference Format:}

Davide Del Vento and Alessandro Fanfarillo. 2019. Traps, Pitfalls and Misconceptions of Machine Learning applied to Scientific Disciplines. In Practice and Experience in Advanced Research Computing (PEARC '19), July 28-August 1, 2019, Chicago, IL, USA. ACM, New York, NY, USA, 8 pages. https://doi.org/10.1145/3332186.3332209

\section{INTRODUCTION}

Fueled by a massive increase increase in hardware capabilities [2], and by small but critical improvements in the algorithms utilized since the late 80s [11], there has been an explosion of Machine

Permission to make digital or hard copies of all or part of this work for personal or classroom use is granted without fee provided that copies are not made or distributed for profit or commercial advantage and that copies bear this notice and the full citation on the first page. Copyrights for components of this work owned by others than the author(s) must be honored. Abstracting with credit is permitted. To copy otherwise, or republish, to post on servers or to redistribute to lists, requires prior specific permission and/or a fee. Request permissions from permissions@acm.org.

PEARC '19, Fuly 28-August 1, 2019, Chicago, IL, USA

(C) 2019 Copyright held by the owner/author(s). Publication rights licensed to ACM ACM ISBN 978-1-4503-7227-5/19/07 ..\$15.00

https://doi.org/10.1145/3332186.3332209 and Deep Learning applications. Understandably, there has been a growing interest by the scientific community in the field, and a few very noteworthy results, such as [7].

On the other hand, we have seen a number of researchers just starting in the field, repeating the mistakes of others. While making mistakes is an effective way to gain familiarity with a new field and learning the tools of the trade, too many mistakes lead to science not done. Therefore, we present here a number of Traps, Pitfalls and Misconceptions of Machine Learning applied to Scientific Disciplines. The focus is mostly, but not exclusively, on Deep Learning, because Deep Learning is the field with the highest interest by the community. To keep the language clear, here are some definitions. By Traps we mean small issues which could be somewhat anticipated and would not cause large problems. On the other hand, by Pitfalls we mean important issues, harder to anticipate and potentially causing large waste of time. Finally, by Misconceptions we indicate ideas or beliefs which could guide the investigations in the wrong direction, also potentially causing very time consuming problems.

The difference between Traps and Pitfalls may be subtle, and it may also depend on the project. Deciding where to put an issue could be debatable. For this paper, one approach could have been to create a single "Traps and Pitfalls" list. However we chose to make the distinction and we are presenting two shorter lists, instead of one, to place greater emphasis on the issues we deem more important, namely the Pitfalls.

This paper is organized as follows. In section 2 we discuss the Misconceptions. In section 3 we cover in details each Pitfall, starting with an in-depth description and then diving into examples, tips and suggestions on how to avoid them. In section 4 we do the same with Traps. Finally in section 5 we state some concluding remarks with a concise summary of the recommended procedures.

\section{MISCONCEPTIONS}

\subsection{Motivation}

In talks, sometimes the motivation for utilizing Machine Learning and/or Deep Learning is said to be trends on Google, or popularity. While these can be good motivations to start studying the field and understand what the hype is about, these are never good ways to motivate a research. An actual motivation analysis must start by asking oneself what problem needs to be solved and what drawbacks current techniques have. This insight will certainly provide a more appropriate choice of tool, ancient or novel, to most effectively tackle the issue. 
It is hard to make broad recommendations regarding this issue, because motivation is a case-by-case factor. In general, however, we suggest the researchers to ask themselves at least the following questions.

(1) If a non-Machine-Learning solution of this problem exists, what aspects of that solution are unsatisfactory, and are hoped to be improved by a Machine Learning implementation?

(a) Accuracy of the results

(b) Speed of the algorithm

(c) Other computing aspects such as memory footprint, or ease of running on a GPU

(d) Other scientific aspects such as trade-off between accuracy and memory/speed of the algorithm

(2) If a non-Machine-Learning solution of this problem does not exist, why not?

(a) The problem is too hard to tackle without Machine Learning, like image or speech recognition, or other "I can tell it, only when I see it" problems

(b) The problem has not been attempted before, but a traditional approach seems feasible

(3) What is one hoping to accomplish with a Machine Learning implementation?

(a) Pedagogic/exploratory goals

(b) Improving any aspect under the question (1)

(c) A faster and/or more agile implementation experience

(4) Is there anybody who has attempted a Machine Learning implementation of this or of a similar problem?

(a) If so, with what success?

(b) If not, why not?

(5) What should success look like, for this project?

Asking oneself these questions will help guide toward making the best choices. For example, answering 2a most likely means the project is a excellent fit for Machine Learning. On the other hand, answering 1a means probably not. Other answers, such as $1 \mathrm{~d}$ or $3 \mathrm{a}$ will depend on the context. In fact, answering $3 \mathrm{a}$ is always good because from the experience something will be certainly learned; however one should consider if the available time and computing power could be spent more effectively on a different Machine Learning project (such as an online class).

\subsection{Names can be misleading}

Machine Learning, Deep Learning and Artificial Intelligence are powerful and fascinating names which can be misleading. While most new technologies often come with expectations that can be unrealistic, how could anything possibly go wrong with an intelligent machine which learns deeply the concepts of the field? We are among the researchers who are not very excited with the choice of these names, and we encourage newcomers of the field to not be mislead by them. These algorithms are very powerful and can be very useful by producing spectacular results. However they are not "magic" and they do not transform computers into Intelligent agents, Learning in the same sense that human beings do, and we urge practitioners to keep it in mind.

\subsection{What Machine Learning is, and what it is not}

Related to the previous misconception is the one that Machine Learning makes the software able to understand the semantic and/or the causation relationships of the problem under study. While there is active research in this topic, e.g. by Pearl [10], the current state of the art is very far from this view. Current Machine Learning algorithms do not model semantic, nor causation.

In fact, Machine Learning are just algorithms like any other. Everything that can be done with Machine Learning, can be done with usual loops, conditionals and traditional numerical methods. The big difference, however, is that with Machine Learning the programmer is freed from having to specify all the logic explicitly with loops, conditionals and math: everything is "automatically" generated by the training data, which effectively becomes "the algorithm".

These observations are important because Machine Learning, and Deep Learning in particular, is able to process very large amount of data and sometimes to "discover" trends, categories, and other information hidden into the data, in a way that could be described as "having insight".

For example, the $k$-means clustering algorithm can partition the observations in $k$ categories, giving the appearance of some "understanding" of what is important in one observation to make it belong to one category, and different from others. In fact, what really happens is simply a partition of the feature space, as sampled by the observations, in $k$ classes, such that each observation belongs to the category with the nearest mean. This is a purely geometric algorithm, and its success on each particular application depends on the practitioner having insights on what the important features are (see Section 3.1), and on what is a good metric for the distance, even though often the regular Euclidean metric works ok, if the dimensionality of the feature space is not too high.

As a another example, one of the first commercial successes of Artificial Intelligence have been Expert Systems which were deployed in the early 1980s (see [12], pag. 24). One important difference between those early Expert Systems and current Machine Learning techniques is that the former needed to have several rules explicitly coded, usually as conditionals, whereas the latter are automatically able to generate that information from the training data. Something in use today that resembles Expert Systems are Decision Trees Learning [9]. In Machine Learning, Decision Trees are a class of algorithms, usually in use for classification problems, which follow several rules (generally conditionals). Unlike Expert Systems rules, Decision Tree ones are generated from data, not from human experts explicitly coding them. There is no "special insight" in how Decision Trees are generated: various algorithms exist, but they all work by doing statistical tests on information gain (i.e. entropy reduction) that can be achieved by splitting the tree in subtrees according to a particular conditional on a particular feature. Both the Decision Tree Learning algorithms, which generate the Decision Trees, as well as the Decision Trees themselves are relatively easy to interpret by humans and therefore misconceptions about them are uncommon. On the other hand, even shallow Neural Networks cannot be easily reasoned on by a humans, because of the cascade of (large) linear operators alternating with the non-linear filters 
- all using apparently random weights. This obfuscation of what happens both during the backpropagation and during the inference in Neural Networks sometimes get simplified to/by newcomers as "magic" especially when terms like Artificial Intelligence and Deep Learning are used to describe the process. In reality, like Decision Trees and Expert Systems do nothing more than apply an algorithm to the input data (conditionals), so Deep Neural Networks do nothing more than applying another algorithm to it (linear algebra and non-linear filters).

\subsection{How Machine Learning compares with Curve Fit}

Most Machine Learning algorithms in use today can be summarized by a two-step process: in the first step some labeled input data (usually large) is used to train the model, and in the second step the trained model is used to make prediction about data which is not "seen" by the model beforehand. This view is obviously ignoring important categories of Machine Learning, such as unsupervised learning, online learning and reinforcement learning.

Viewing Machine Learning as the two-step process described above, its similarity with curve fitting is striking: both techniques are used to find a function from the feature space to the label space (either classification or regression) which approximate "best" the labeled input data, while at the same time having "good chances" of predicting the value for unseen points.

Even when this view holds perfectly, it is sometimes used or understood in a derogative or dismissive attitude. We would like to be clear that we do not intend it in such a way. We are using it in a pure descriptive fashion, finding difference and similarities to help our thought process, and the one of other researchers. Moreover, the view does not always hold perfectly, for example because curve fitting is practically always intended for function of continuous variables, and not too many of them, whereas Machine Learning does not make those assumptions, and in fact it is often applied to an extremely large number of variables (for example the pixels of an image) allowed to be set to a small or large number of discrete values (for example a small discretized number of luminance values).

One similarity between Machine Learning and Curve Fitting is that the practitioner needs to make choices: What kind of curve one wants to use? Polynomial? If so, what degree? With how many parameters? The latter question is particularly important: a curve fitting with a large number of parameters can reduce the error on the training set to an arbitrarily small value. However, that may not capture the actual underlying phenomenon, and may fail to predict subsequent values which are not in the training set. The same situation applies to Machine Learning and is usually described as "failure to generalize". Both in curve fitting and in Machine Learning, this can happen for a variety of reasons, for example inaccuracies (or "noise") in the training sets. The strategies to overcome the issue are different: in curve fitting one uses the Occam's razor and some understanding of the underlying phenomenon being modeled, whereas in Machine Learning there are various approaches, depending on what the root cause. Such approaches will be described in Sections 3 and 4

As a practical example, consider increasing the number of layers in a Neural Network, without changing anything else. This will increase its degrees of freedom - somewhat similar to increasing the degree of a polynomial used to fit some points. Could this increase of the number of layers improve the performance of the Neural Network? It could, if the reason for poor performance is that the model of the less deep network did not have enough degrees of freedom and if there is enough labeled data to determine the additional parameters. As a rule of thumb, for a dense network this is seldom the case, however for a relatively shallow convolutional network this is more often possible. As a different example, consider fitting an exponential curve with a polynomial function. Since the number of data points to fit cannot be infinite, this is clearly possible, and increasing the degree of the polynomial can increase accuracy, at least to some level. However, if the practitioner has the insight to recognize that a different curve is a better fit, the accuracy will increase much more with much less effort. Having this insight can be trivial for a function of single variable, relatively easy for a function of a limited number of variables, but can be difficult for a function with lots of variables and a very intricate behavior against each one of them. Visualizing and finding intuition in a high dimensional, involved hyperspace is not easy. The same difficulties apply to Machine Learning and to Deep Learning in particular: the choice to add further Convolutional layers or Pooling layers, and how many of them, and where in the architecture of the network, could change the performance dramatically. In the subsequent sections we will suggest some tips on how to make this kind of choices.

\subsection{How Machine Learning compares with a Statistical Model}

Machine Learning is also similar to Statistical Models: they both try to model the underlying distribution of the known (training) data, to make predictions for the data which is not known. However, they have been developed by different communities, they make different assumptions, they require different tools, and they existed for very different time periods. In particular, Statistical Models have been developed by mathematicians for quite a long time, often in very rigorous frameworks, considering detailed assumptions on the data and only drawing certain conclusions. On the other hand, Machine Learning models have been developed mostly by computer scientists for just a few decades, making very little assumptions on the data and drawing conclusions which may or may not be rigorously accurate. The most remarkable difference is that Machine Learning rely on larger amount of data: even for small Machine Learning projects, data drives the model and therefore an adequate volume is needed; in Statistical Models the assumptions made on the distributions of the data allow the practitioners to draw conclusions from potentially much smaller volumes.

\section{PITFALLS}

\subsection{The features are not meaningful or well constructed}

Machine learning algorithms, and in particular Neural Networks, operate on numerical (usually floating point) variables. A good feature for a machine learning problem should always have a meaningful magnitude to help the algorithm making good predictions. 


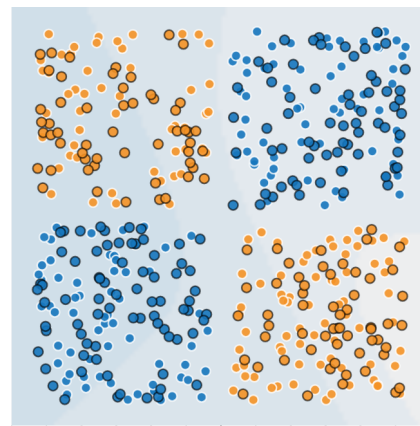

Figure 1: Raw data

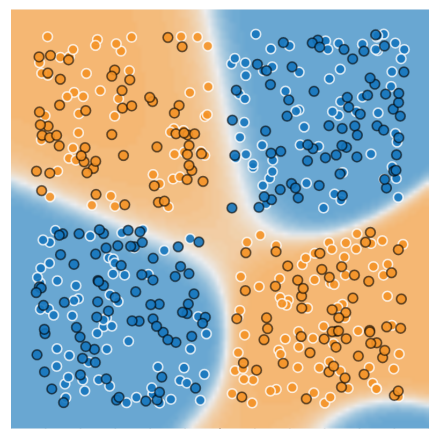

Figure 2: Classification by a small Neural Network using raw features

Raw data fed straight to a machine learning algorithm rarely produce decent results.

As an example, consider the classification problem showed in Figures 1-4. The labeled data points are represented by colored circles. The ones with white border are used for training, and the ones with black for testing. Orange corresponds to positive examples and blue to negative ones. In Figure 1 only the labeled data is presented. On the $x$ and $y$ axes are two features which we refer to as $x_{1}$ and $x_{2}$. In Figures 2-4 the classification from three different Neural Networks are in the background under the labeled data, using the same color scheme. The figures for this section have been generated with [1] The classification of Figure 2 has been obtained from a relatively small Neural Network with 2 hidden layers and 5 total units. Trying to improve the accuracy with hyperparameter tuning did not yield much of an improvement, however increasing the model size did, as shown in Figure 3. This second Neural Network had 3 hidden layers and 8 total units. It has been possible to have the larger network to converge to decent results, however that required careful tuning of the hyperparameters. On the other hand, creating a simple engineered feature, and adding it to the input features of a tiny Neural Network, yielded the results shown in Figure 4. This last network had only a single layer, and only 2 units. Yet, it provided the best accuracy and its performance have been very stable and not overly depending on careful tuning of hyperparameters. This remarkable result has been possible by engineering a single, new feature using the "insight" that the two features had a relationship which could

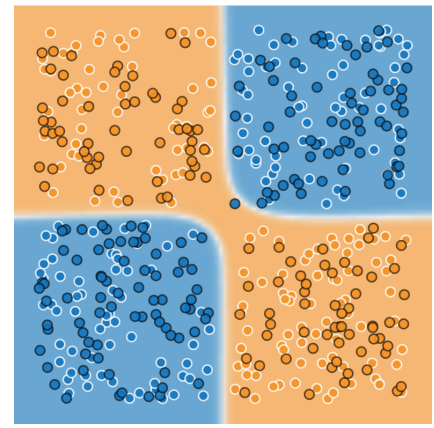

Figure 3: Classification by a larger Neural Network using raw features

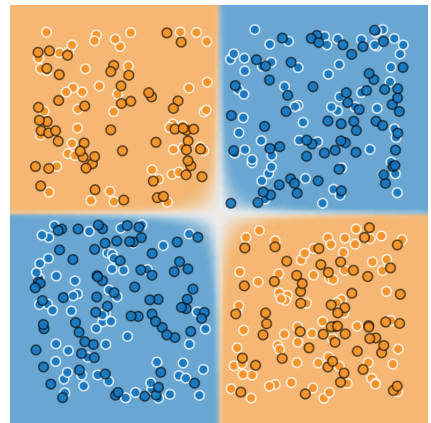

Figure 4: Classification by a tiny Neural Network using engineered features

be exposed more clearly to the model by crossing them by a simple multiplication:

$$
x_{3}=x_{1} * x_{2}
$$

The act of extracting features from raw data and transforming them into formats that are suitable for machine learning model is called feature engineering and represents one of the most important task in a machine learning project. Examples of feature engineering tasks are: feature scaling, outliers removal, features encoding and crossing.

\subsection{Bias-Variance tradeoff, Overfitting and Underfitting}

All but the most simple Machine Learning algorithms have a number of hyperparameters to be chosen, and the most powerful techniques have a large number. In Deep Learning one has to select the number of layers, the number of hidden units in each layer, the kind of layers to be used (for example if a convolutional, a pooling or a fully connected layer) and their hyperparameters (for example, in a convolutional layer, the size of the receptive field), what to use for activation function, what value of learning rate to use, and more. It is very unlikely that even experts are able to select the most appropriate values of hyperparameters at first try, especially when exploring something new, which is what researchers do in Scientific Disciplines. 


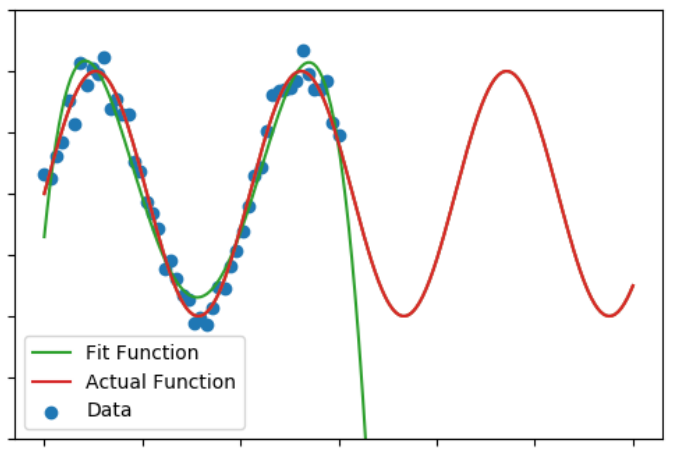

Figure 5: Polynomial fit of a periodic function

In fact, applying Machine Learning successfully is a highly iterative process: practitioners decide what to utilize, for example Deep Learning, configure and run the model, assess the performance, then tune a plethora of hyperparameters, and repeat the configurerun-assess-tune cycle over and over - until the desired performance are achieved. It is essential that during this process the researchers get appropriate feedback to adjust the settings in the right direction, because a random search in the highly dimensional space of the hyperparameters is unfeasible (even though it is often possible and sometimes it is the best approach for exploring small parts of the subspace). Moreover, it is also highly desirable that the loop runs as fast as possible, so that one sees the results of the changes almost immediately, and the thought process is not interrupted by waiting for the results to come.

For this purpose, understanding the related concepts of Bias, Variance, Overfitting and Underfitting is essential. In addition, it is very important to know what to do when each one of these is identified, to move forward in the iterative cycle described above and knowing what to do is the purpose of this section. Most of the information about this topic has been taken from [3].

\subsubsection{What are Bias, Variance, Overfitting and Underfitting}

In Machine Learning (and Statistics), the bias is an error of the model that results from not capturing (or not fully capturing) the relationship between the the features and the response variable that is being predicted. It is usually the consequence of not using a correct model, hence the name bias. Following our own suggestion described in Section 2.4, we guide our thoughts using a curve fit: for example, trying to fit a phenomenon which has a periodic behavior (say sinusoidal, see Figure 5) with a polynomial function will result in a high error, because it is impossible for the fit function to correctly approximate the behavior of the actual function in a wide enough range of the domain, especially when extrapolating. In Machine Learning, a situation like this is called underfit.

On the other hand, the variance is an error of the model that results from capturing too much from the training set. It also can be the consequence of not using the correct model, but usually in

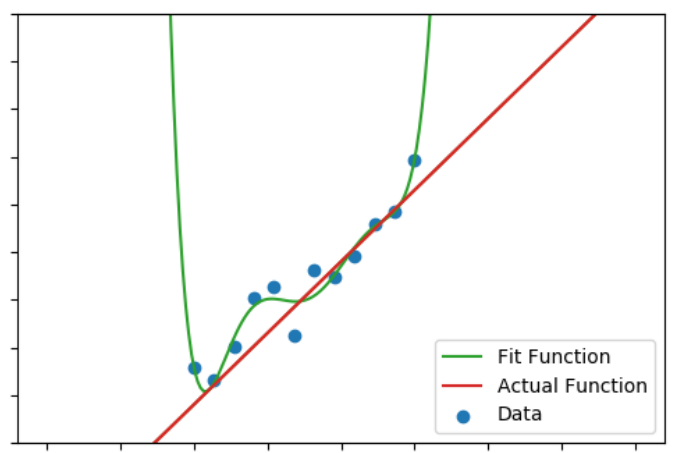

Figure 6: High-degree polynomial fit of a linear function

opposite sense of having too many degrees of freedom and modeling the noise in the data, rather than the actual phenomenon of interest. For an example similar to the one regarding bias, one may be trying to fit a phenomenon which has a low-degree polynomial behavior with a high-degree function (Figure 6). The high-degree polynomial is sensitive to the inevitable noise present in the training data. In this way, the fit function "moves too much" hence the name variance. More than the unnecessary ups-and-downs, the problem with variance is that the fit likely fails to correctly extrapolate, as Figure 6 shows. In Machine Learning, this very situation is called overfit. Notice as for both bias and variance the fit can be particularly poor when extrapolating.

In general, to decrease the bias (in Curve Fitting as well as in Machine Learning) one has to utilize a more general model, but if the model is too general one incurs a high risk to increase the variance. In low dimensionality problems it is possible to get the model "just right", as depicted in Figure 7 for a curve fit. However, as the dimension of the problem increases, that becomes harder and harder, even in curve fit. Sticking to a polynomial fit as an example, it is possible that some of the predictor variables need a high degree polynomial, whereas others need a low degree one, complicating a lot the search for the "just right" degree. When one considers the extremely high dimensionality of most Machine Learning problems, and the further possibility of Neural Network to model very sophisticated functions with meandering behavior, finding the correct bias-variance trade-off seems hopeless. And in fact, with traditional approaches, it has always been a very hard nut to crack However, recent techniques have been devised in Deep Learning to defy the bias-variance trade-off. Before getting there, though, we need to discuss how to split the labeled dataset.

\subsubsection{Best approaches in splitting the labeled dataset.}

One pitfall which could be mistaken for an almost irrelevant detail is utilizing the $k$-fold or the leave-one-out cross-validation approach, instead of a more modern one. In fact, it has been (and still is) common to split the labeled dataset in two sets: one for training, the other for validating, often in $70 \%-30 \%$ or $80 \%-20 \%$ ratios. The purpose of such a split is to have some extra data, after the model 
has been trained, to assess its performance on unseen data, in order to get an estimate of performance in production. One problem of this approach in "small data" Machine Learning research is the reduction of the already limited training data. A second, even more important, problem is the fact that when poor performance are identified on the validation set, one has to change something into the model and repeat the process, often several times - resulting in virtually fitting the model to the validation set, and risking to fall into the high variance problem described above. To partially mitigate these problems, leave-one-out cross-validation or $k$-fold cross-validation were commonly used. However these techniques do not really solve the problem at the root. Moreover, with this approach, there is no good guidance in what exactly change when poor performance are identified.

The alternative splitting strategy we recommend is to split the labeled dataset in three, rather than two sets. One of these sets is for training (train), another is held out for cross-validation (dev), and the last one is for testing (test). The train and dev sets are used almost in the same way that the old train and validation sets are, with repeated iterations trying to optimize performance. However the test set is used very seldom, ideally only once or a handful of times per project, and therefore no fitting to the test set can occur. Instead of splitting to a fixed percentage of the available labeled datasets, we suggest to use a count of items. For example, if one is fortunate enough to work on a Big Data problem with millions of labeled data points, $1 \%$ (or less) for each of the dev and test sets suffice, amounting to tens of thousands of data points. One very important issue to know is that the train, dev and test sets should come from the same distribution. However sometimes that is hard to achieve. If so, it is essential that the dev and test do, even if the distribution of the training dataset is different. This is common, for example, in image processing: often in Scientific Disciplines researchers do not have the huge number of images needed to train the Deep Neural Networks necessary for high performance image recognition. However starting from models pre-trained on common images of cats is often adequate, as long as the dev and test sets are instead coming from the actual distribution of interest, e.g. of satellite images.

\subsubsection{Identifying Bias and Variance problems to defy the tradeoff.}

The first step for starting a Machine Learning project, once one has done the preliminary data preparation as discussed in the Sections 3.1 and 3.2.2, is to pick up a model to utilize. Suppose, for the sake of the argument, that the researcher decides to use a Deep Learning model.

After that, the configure-train-assess-tune cycle start. At first, the configure step will be selecting some reasonable defaults. The training step will produce a model which, at least initially, will probably not model the training data well enough. The first obvious observation is that if one cannot fit the training set, there is no point in doing anything else. Usually researchers are able to reduce the bias and fit the training set without large efforts or using our tip, however for the sake of being complete here is the first one.

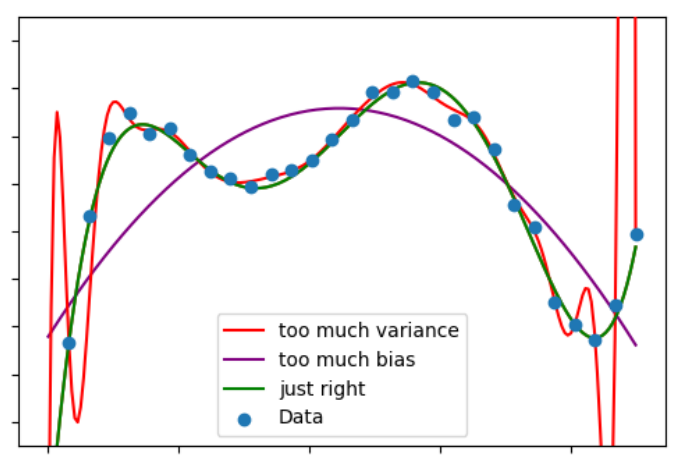

Figure 7: Bias-Variance tradeoff for curve fit

TIP 1. High error on the training sets (underfitting) means High Bias

- Try bigger network (i.e. more layers and/or more hidden units)

- Increase the number of training iterations

- Use a more advanced optimization algorithm (like Adam or momentum)

- As a last resort, use a different architecture

In most cases, Deep Neural networks have enough degrees of freedom that the suggestions of Tip 1 are unnecessary, however we are being explicit because we would like to highlight an important point: the information one would like to extract from the data may not be there. As an extreme example, consider a researcher who is attempting to identify heat waves in a large dataset of climate data which (for the sake of the argument) does not contain temperature, but has labels for heat-wave or not-heat-wave events. It will be extremely difficult, if not impossible, to identify if a given area on a given day is experiencing a heat wave without knowing the temperature! Yet, there might be other indicators, such as wind speed/direction or lack of precipitation, which could be correlated with a heat wave, and the Deep Learning model could find them. If the correlation between heat waves and these other indicators is imperfect, it would be impossible to exactly fit the training set. This is the reason why we are generically saying "high error" without actually specifying a value. What "high" means will be clear after the next tip.

Another remark about trying a bigger network by manually selecting more layers and/or more hidden units. This is certainly something that can be explored, however what exactly to do is more of an art than a science. For Deep Learning newcomers, people with limited time, and/or limited amount of labeled data (all common among practitioners of Scientific Disciplines) this tip is best interpreted as find an existing bigger network in the literature which has been documented having good performance on a similar problem. For example, for satellite image recognition one could start from LeNet [8], them move to AlexNet [6] or even ResNet[4]. For a problem which involves sequences more than geometric image-like structures, such as seismic analysis, one could experiment with 
Long Short-term Memory architecture [5] and possibly add concepts like "attention" [13] if necessary.

Once the performance on the training set are deemed sufficient, one can move forward in the cycle, and assess the performance on the dev set set. Likely, at least at first, there will be a high error on such a set, which leads to our second tip.

TIP 2. High error on the dev set (overfitting) means High Variance

- Find more labeled data

- Use a regularization technique such as $L_{2}$ or dropoff

- As a last resort, try a different architecture

Having split the dataset in three allows one to use the dev set to immediately identify overfitting, take one of the corrective actions suggested in Tip 2 and carry on the iterative process - without worrying about the risk of fitting the model to the dev set. Here too, we have been generic about "High error", for the same reason mentioned above. However now we can clarify what we mean by "High error" in both cases. By "error" here we mean the the percentage of mislabeled examples in the relevant set (or of examples with errors greater than an acceptable threshold for regression). If the error is less than (say) $5 \%$, for most purposes it can be considered small. However, if one requires better accuracy than 5\% percent and has enough good quality training data, it is totally fine to use a smaller threshold. If the error on the dev set is more than 5\% (or whatever the chosen threshold it), it is important to compare it with the error on the train set: they could be of the same order, or one could be much larger than the other (say at least $2 \mathrm{x}$ or more):

- If the error on the dev set and the one on the train set are of the same order, it is possible to be in a situation of Underfitting, so it is important to evaluate if that is the case, and apply the Tip 1 . In fact, if some information is missing from the data (as in the example of the heat wave above), the model is probably working just fine. In such a case, the researcher has to accept the achieved level of error as the best that can be done, or figure out which information needs to be added to the data, and add it, if possible.

- If the train error is larger than the dev error, it is likely that the researcher is in front of a spurious correlation. This is common with small datasets, for which the statistics make it not rare. If possible, increasing the size of the dev set is recommended. If that is not possible because the number of labeled examples is limited, one could carry on but should be wary of the fluke and not expecting errors lower than the ones achieved during training.

- If the train error is smaller than the dev error, then it is definitely a case of Overfitting and Tip 2 should be applied before proceeding with the iterations.

At this point, the research would need to iterate among Tip 1 and Tip 2 until the error becomes acceptable. Applying the suggestions above is best, because they tend to affect almost exclusively the problem which is being addressed. On the other hand, other techniques, such as Early Stopping during the training, affect both the error on the training set and the one on the dev set, causing the need for a bias-variance tradeoff.
Finally, when the researchers are satisfied with the level of errors for both the training and dev set, they should assess performance on the test set. As explained above, it is important to not do that too many time (one time only would be ideal). If a low error is achieved on the test set, then the cycle is complete. For "low" here we mean of the same order of the error on the dev set (or of the train set if the fluke described above had been experienced). Sometimes this is not the case, and a high error on test set is found. For such a case we have the last tip of this section.

\section{TIP 3. High error on the test sets}

- Restart from scratch with a larger dev set, or

- Restart from scratch with a different cost function

The rationale for this last tip is that the model is failing to generalize, and this could be due to one of two reasons: the Neural Network has been fit to the test set, or it is not correctly modeling the phenomenon.

When can one conclude that the train/dev/set have not been extracted from the same distribution and therefore one of more of those sets should be changed?

\section{TRAPS}

The Traps are the issues which we deem minor, but still worth enough attention and consideration.

\subsection{The data is too noisy}

In some circles, Machine Learning is building a reputation for being too sensitive to "noise" in the training data. This is by design, to most accurately fit the training set, as already mentioned in Section 2. However, in many cases, that is not what the goal of the research is. Removing outliers and performing some sort of scaling mitigates the effect of the noise. Furthermore, especially if the noise is not very severe, and/or if the dataset is large enough, the model performance may be affected, but the overall results can still be acceptable and hence we are classifying it as a trap rather than a pitfall.

\subsection{Not enough data is available}

Most, but not all, Machine Learning algorithms really thrive when they are fed an enormous amount of data, as shown in Figure 8 This is certainly true for Deep Learning (however tiny neural networks with a small number of neurons/connections can in some cases produce good result with limited amount of data).

Selecting the Machine Learning algorithm most appropriate for the problem at hand and the amount of available data is the easy path out of this trap.

\subsection{Using Machine Learning instead of known explicit equations}

Often times, we have seen newcomers applying Machine Learning to problems which had known explicit equations governing the phenomenon. While this is not erroneous per se, sometimes there is blind hope to get a solution with less compute time and/or with more accuracy. This is seldom the case and when it does happen it is 


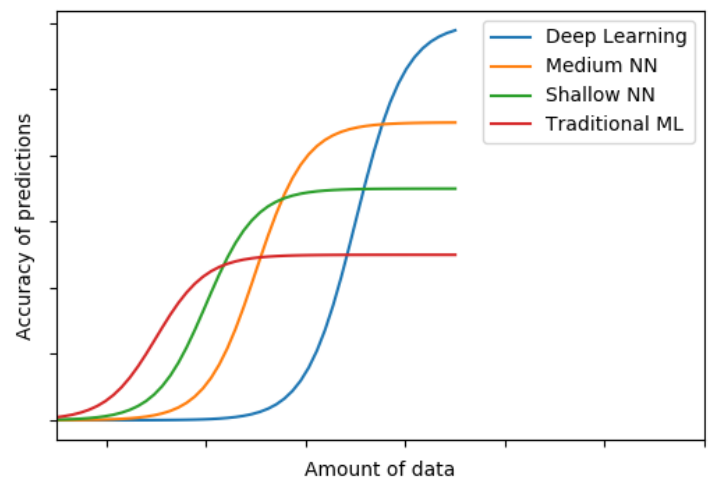

Figure 8: Qualitative performance comparison of various Machine Learning techniques

usually because the phenomenon has an emergent behavior which is difficult to reproduce from the equations. Moreover, caution must be exercised to expose the Machine Learning models to fully representative data, especially if there are thresholds above/below which something changes, as it often happens in Chemistry on in fields involving a phase change.

On the other hand, exploring a known problem with a solution known independently can certainly help from a pedagogic perspective, so in that context using Machine Learning to model a phenomenon governed by known explicit equations can be very useful.

\subsection{Trying over and over until it works}

Given the iterative nature of the Machine Learning techniques, it is normal to try different parameter, settings, architectures, and more, until the "right" ones are found. However, it is important to do so with statistical consistency, rigor and a grain of salt. One must be aware of spurious correlations like the famous one between Divorce Rate in Maine and Per-capita Consumption of Margarine in the US By trying over and over, it is not uncommon to find something that works "by accident" for the train set, perhaps for the dev set, and sometimes even for the test set - especially when the size of the various sets are small. In such a case, a failure to generalize on real world data is the conclusion.

\section{SUMMARY}

- Start with a strong motivation analysis before embarking in a new project

- Not be misled by names

- When appropriate, think of Machine Learning as a curve fit and/or a statistical model, and consider its degrees of freedom

- Use scientific insight about which features to use

- Split the labeled dataset in three: train, dev and test sets

- Make sure train, dev and test sets come from the same distribution (or, if that is not possible, that at least the dev and test sets come from the same distribution)
- Decide what model and architecture to use and get into the configure-train-assess-tune cycle

- Train on the training set and assess the performance on that set, until the results are satisfactory. For Deep Learning, see Tip 1.

- When performance on the training set are satisfactory, assess them on the dev set, until the results are satisfactory. For Deep Learning, see Tip 2.

- When performance on the dev set are satisfactory, assess them on the test set, until the results are satisfactory. Be wary of using the test set more than a handful of times! For Deep Learning, see Tip 3.

\section{ACKNOWLEDGEMENTS}

This material is based upon work supported by the National Center for Atmospheric Research, which is a major facility sponsored by the National Science Foundation under Cooperative Agreement No. 1852977.

\section{REFERENCES}

[1] [n. d.]. Tensorflow playground. https://github.com/tensorflow/playground. Accessed: 2019-04-10.

[2] T. Baji. 2018. Evolution of the GPU Device widely used in AI and Massive Parallel Processing. In Proceedings of the 2nd IEEE Electron Devices Technology and Manufacturing Conference (EDTM). https://doi.org/10.1109/EDTM.2018.8421507

[3] Ian Goodfellow, Yoshua Bengio, and Aaron Courville. 2016. Deep Learning. MIT Press. http://www.deeplearningbook.org.

[4] Kaiming He, Xiangyu Zhang, Shaoqing Ren, and Jian Sun. 2015. Deep Residual Learning for Image Recognition. CoRR abs/1512.03385 (2015). arXiv:1512.03385 http://arxiv.org/abs/1512.03385

[5] Sepp Hochreiter and JÃijrgen Schmidhuber. 1997. Long Short-Term Memory. Neural Computation 9, 8 (1997), 1735-1780. https://doi.org/10.1162/neco.1997.9. 8.1735 arXiv:https://doi.org/10.1162/neco.1997.9.8.1735

[6] Alex Krizhevsky, Ilya Sutskever, and Geoffrey E. Hinton. 2017. ImageNet Classification with Deep Convolutional Neural Networks. Commun. ACM 60, 6 (May 2017), 84-90. https://doi.org/10.1145/3065386

[7] Thorsten Kurth, Sean Treichler, Joshua Romero, Mayur Mudigonda, Nathan Luehr, Everett Phillips, Ankur Mahesh, Michael Matheson, Jack Deslippe, Massimiliano Fatica, Prabhat, and Michael Houston. 2018. Exascale Deep Learning for Climate Analytics. In Proceedings of the International Conference for High Performance Computing, Networking, Storage, and Analysis (SC 2018). IEEE Press, Piscataway, NJ, USA, Article 51, 12 pages. http://dl.acm.org/citation.cfm?id=3291656.3291724

[8] Yann Lecun, LÃlon Bottou, Yoshua Bengio, and Patrick Haffner. 1998. Gradientbased learning applied to document recognition. In Proceedings of the IEEE. 22782324.

[9] T.M. Mitchell. 1997. Machine Learning. McGraw-Hill.

[10] Judea Pearl. 2004. Robustness of Causal Claims. In Proceedings of the 20th Conference on Uncertainty in Artificial Intelligence. AUAI Press, Arlington, VA, 446-453.

[11] W. Rawat and Z. Wang. 2017. Deep Convolutional Neural Networks for Image Classification: A Comprehensive Review. Neural Computation 29 (2017). Issue 9. https://doi.org/10.1162/neco_a_00990

[12] Stuart Russell and Peter Norvig. 2010. Artificial Intelligence: A Modern Approach (3rd ed.). Pearson Education, Inc., Upper Saddle River, New Jersey 07458.

[13] Ashish Vaswani, Noam Shazeer, Niki Parmar, Jakob Uszkoreit, Llion Jones, Aidan N Gomez, Ł ukasz Kaiser, and Illia Polosukhin. 2017. Attention is All you Need. In Advances in Neural Information Processing Systems 30, I. Guyon, U. V. Luxburg, S. Bengio, H. Wallach, R. Fergus, S. Vishwanathan, and R. Garnett (Eds.). Curran Associates, Inc., 5998-6008. http://papers.nips.cc/paper/ 7181-attention-is-all-you-need.pdf 